\title{
Inverse analysis of forced convection in micro-channels with slip flow via integral transforms and Bayesian inference
}

\author{
Carolina P. Naveira-Cotta, Renato M. Cotta*, Helcio R.B. Orlande \\ Laboratory of Transmission and Technology of Heat - LTTC, Mechanical Engineering Dept. - COPPE E Escola Politécnica, Universidade Federal do Rio de Janeiro,
} Cx. Postal 68503, Cidade Universitária Rio de Janeiro, RJ 21945-970, Brazil

\section{A R T I C L E I N F O}

\section{Article history:}

Received 24 August 2009

Received in revised form

14 December 2009

Accepted 17 December 2009

Available online 6 February 2010

\section{Keywords:}

Micro-channel

Micro-scale heat transfer

Inverse problem

Integral transforms

Bayesian inference

Accommodation coefficients

\begin{abstract}
A B S T R A C T
The present work addresses the direct and inverse problems for convective heat transfer with incompressible laminar gas flow in micro-channels, within the range of validity of the slip-flow regime. The direct problem analysis combines the classical integral transform method and the generalized integral transform technique (GITT), by analytically solving the two-dimensional steady-state convection problem and finding a hybrid numerical-analytical solution for the required eigenvalue problem. The inverse problem analysis makes use of the accuracy and robustness of the direct problem solution and focus on the simultaneous identification of the momentum and thermal accommodation coefficients, related to gas flow and heat transfer within micro-channels, besides the usually unknown boundary condition parameters, here represented by the external Biot number. The inverse analysis is based on the availability solely of temperature measurements at the channel external wall, along its length, as obtained for instance via infrared camera thermography. A Bayesian inference approach is adopted in the solution of the identification problem based on the Monte Carlo Markov Chain method (MCMC) and the Metropolis-Hastings sampling algorithm. A typical example of slip flow in parallel-plates micro-channel is selected to illustrate both the direct and inverse problems solution approaches.
\end{abstract}

() 2009 Elsevier Masson SAS. All rights reserved.

\section{Introduction}

The analysis of internal flows in the slip-flow regime gained an important role along the last two decades in connection with microelectromechanical systems (MEMS) applications and in the thermal control of microelectronics, as reviewed in different sources [1-5]. Several steady-state incompressible flow situations in laminar regime within simple geometries, such as circular micro-tubes and parallel-plate micro-channels, developed for the slip-flow regime, have been widely employed in the heat transfer analysis of microsystems [6,7]. Also recently in [8-12], the analytical contributions were directed towards more general steady and transient problem formulations, including viscous dissipation, axial diffusion in the fluid and three-dimensional flow geometries.

In this context, the first goal of this paper is thus to illustrate the results obtained from a fairly general hybrid numerical-analytical solution for temperature distributions in a fluid flowing through two- or three-dimensional micro-channels, taking into account the velocity slip and temperature jump at the walls surfaces. For this purpose, a flexible approach was employed [13], based on formal

\footnotetext{
* Corresponding author. Tel.: +55 212562 8567; fax: +55 2125628383 .

E-mail address: cotta@mecanica.coppe.ufrj.br (R.M. Cotta).
}

solutions of the energy equation as obtained via the classical integral transform method [14], in association with the Generalized Integral Transform Technique, GITT [15-18], which was used for the solution of the required eigenvalue problem [19-21]. This method is here applied for illustration purposes in the integral transformation of the energy equation for thermally developing flow within parallel-plates micro-channels under the slip-flow regime. This combination of solution methodologies provides a very effective eigenfunction expansion solution, through the fast converging analytical representation in all the space coordinates, together with a flexible and reliable numerical-analytical approach for the SturmLiouville eigenvalue problem solution.

The accuracy of such analytic-type solutions for the direct forced convection problem in micro-channels are however dependent on the also accurate determination of the momentum and thermal accommodation coefficients, as required by the slip and temperature jump boundary conditions inherent to the slip-flow model that accounts for non-continuum effects at the fluid-surface interactions. Fundamental experimental work on rarefied gas dynamics have offered measurements of the tangential momentum accommodation coefficient, requiring, for instance, high vacuum and molecular beams impinging on carefully prepared substrates, such as recently reviewed in [22], but very few results are available for the actual conditions of the flow 


\begin{tabular}{|c|c|c|c|}
\hline \multicolumn{2}{|c|}{ Nomenclature } & \multirow{3}{*}{$\begin{array}{l}y_{1} \\
z, Z\end{array}$} & \multirow{2}{*}{$\begin{array}{l}\text { half spacing between parallel plates } \\
\text { dimensional and dimensionless longitudinal } \\
\text { coordinate, respectively }\end{array}$} \\
\hline $\mathrm{Bi}$ & Biot number & & \\
\hline$d(\mathbf{x})$ & linear dissipation operator coefficient, eq. (1a) & & \\
\hline$I$ & number of parameters to be estimated & \multicolumn{2}{|c|}{$\alpha(\mathbf{x}), \beta(\mathbf{x})$ boundary condition coefficients, eq. (1c) } \\
\hline$k(\mathbf{x})$ & diffusion operator coefficient, eq. (1a) & $\alpha$ & fluid thermal diffusivity \\
\hline Kn & Knudsen number $\left(=\lambda / 2 y_{1}\right)$ & $\alpha_{m}, \alpha_{t}$ & tangential momentum and thermal accommodation \\
\hline$N_{i}$ & normalization integrals & $\beta_{t}$ & temperature jump coefficient, eq. (6e) \\
\hline $\mathrm{Nu}(Z)$ & local Nusselt number & $\beta$ & boundary condition coefficients ratio, $\beta_{t} / \beta_{v}$ \\
\hline $\mathbf{P}$ & vector of unknown parameters & $\phi(\mathbf{x}, t)$ & equation source term, eq. (1a) \\
\hline$P(\mathbf{x}, t)$ & equation source term, eq. (1a) & $\lambda$ & molecular mean free path \\
\hline $\operatorname{Pr}$ & Prandtl number & $\lambda_{n}$ & eigenvalue of auxiliary problem \\
\hline$u(y)$ & velocity field inside channel & $\Theta$ & vector of estimated temperatures \\
\hline$u_{a v}$ & average flow velocity & \multirow{2}{*}{\multicolumn{2}{|c|}{ Subscripts $\mathcal{E}$ Superscripts }} \\
\hline$w(\mathbf{x})$ & transient operator coefficient, eq. (1a) & & \\
\hline$W(Y)$ & dimensionless velocity profile & $i, n, m$ & order of eigenquantities \\
\hline $\mathbf{W}$ & covariance matrix of the measurement errors & - & integral transform \\
\hline $\boldsymbol{x}$ & position vector & $\sim$ & normalized eigenfunction \\
\hline$y, Y$ & $\begin{array}{l}\text { dimensional and dimensionless transversal } \\
\text { coordinate, respectively }\end{array}$ & & \\
\hline
\end{tabular}

configuration within micro-channels and their actual bounding walls $[23,24]$. The experiments indicate that the tangential momentum accommodation coefficient generally assumes values between 0.2 and 1.0, with the lower limit being associated with exceptionally smooth surfaces and the upper limit with very rough or highly oxidized surfaces [4]. Similar considerations are pertinent to the measurement of thermal accommodation coefficients [25], where an even more limited experimental database is available, and apparently no previous work seems to be available on the identification of this coefficient in actual heat and fluid flow conditions within specific pressure and temperature levels pertinent to MEMS applications, and in addition for actual morphology and finishing of the micro-channel walls.

Thus, we take advantage of the accuracy, robustness and efficiency of the direct problem solution, to tackle the associated inverse heat transfer problem analysis $[26,27]$ towards the simultaneous estimation of momentum and thermal accommodation coefficients in microchannel flows with velocity slip and temperature jump. A Bayesian inference approach is adopted in the solution of the identification problem, based on the Monte Carlo Markov Chain method (MCMC) and the Metropolis-Hastings algorithm [28-31]. Only simulated temperature measurements at the external faces of the channel walls, obtained for instance via infrared thermography [31], are used in the inverse analysis in order to demonstrate the capabilities of the proposed approach. A sensitivity analysis allows for the inspection of the identification problem behavior when the external wall Biot number is also included among the parameters to be estimated.

\section{Analysis}

\subsection{Direct problem solution}

The approach here employed in the direct problem solution for forced convection in micro-channels, is borrowed from a recent work on diffusion in heterogeneous media, with arbitrarily space variable thermophysical properties [13]. In this sense, the dimensionless velocity fields are mathematically equivalent to space variable thermal capacitances, and the formal solution procedure is here briefly described.

We consider a general formulation on steady or quasi-steady state hydrodynamically fully developed and thermally developing convection, that governs the temperature field $T(\mathbf{x}, z)$, dependent on the position $\mathbf{x}$ on the transversal plane and on the longitudinal coordinate $z$, defined in the transversal region $\mathrm{V}$ with boundary surface $S$. The formulation includes the convection term, the diffusion operator, a linear dissipation term, and an independent source term [13], as shown in problem (1) below. The coefficients $w(\mathbf{x}), k(\mathbf{x})$, and $d(\mathbf{x})$, are responsible for the information related to the velocity field, geometry and eventually even heterogeneity of the medium. The general formulation is given by the convection-diffusion equation and inlet and boundary conditions below:

$$
\begin{aligned}
& \begin{aligned}
w(\mathbf{x}) \frac{\partial T(\mathbf{x}, z)}{\partial z}= & \nabla \cdot k(\mathbf{x}) \nabla T(\mathbf{x}, z)-d(\mathbf{x}) T(\mathbf{x}, z) \\
& +P(\mathbf{x}, z), \mathbf{x} \in V, z>0
\end{aligned} \\
& T(\mathbf{x}, 0)=f(\mathbf{x}), \quad \mathbf{x} \in V, \\
& \alpha(\mathbf{x}) T(\mathbf{x}, z)+\beta(\mathbf{x}) k(\mathbf{x}) \frac{\partial T(\mathbf{x}, z)}{\partial \mathbf{n}}=\phi(\mathbf{x}, z), \quad \mathbf{x} \in S
\end{aligned}
$$

The exact solution of problem (1) is obtained with the Classical Integral Transform Method [14,15], and is written as:

$T(\mathbf{x}, z)=\sum_{i=1}^{\infty} \tilde{\psi}_{i}(\mathbf{x})\left(\bar{f}_{i} e^{-\mu_{i}^{2} z}+\int_{0}^{z} \bar{g}_{i}\left(z^{\prime}\right) e^{-\mu_{i}^{2}\left(z-z^{\prime}\right)} d z^{\prime}\right)$ 
where the eigenvalues $\mu_{i}$ and eigenfunctions $\psi_{i}(\mathbf{x})$, are obtained from the eigenvalue problem that contains the information about the velocity field, in the form:

$\nabla \cdot k(\mathbf{x}) \psi_{i}(\mathbf{x})+\left(\mu_{i}^{2} w(\mathbf{x})-d(\mathbf{x})\right) \psi_{i}(\mathbf{x})=0, \quad \mathbf{x} \in V$

$\alpha(\mathbf{x}) \psi_{i}(\mathbf{x})+\beta(\mathbf{x}) k(\mathbf{x}) \frac{\partial \psi_{i}(\mathbf{x})}{\partial \mathbf{n}}=0, \quad \mathbf{x} \in S$

Also, the other quantities that appear in the exact solution (2) are computed after solving problem (3), such as:

$N_{i}=\int_{V} w(\mathbf{x}) \psi_{i}^{2}(\mathbf{x}) \mathrm{d} v$, normalization integrals,

$\tilde{\psi}_{i}(\mathbf{x})=\frac{\psi_{i}(\mathbf{x})}{\sqrt{N_{i}}}, \quad$ normalization eigenfunctions

$\bar{f}_{i}=\int_{V} w(\mathbf{x}) \tilde{\psi}_{i}(\mathbf{x}) f(\mathbf{x}) d v, \quad$ transformed inlet conditions

$\bar{g}_{i}(z)=\int_{V} P(\mathbf{x}, z) \tilde{\psi}_{i}(\mathbf{x}) d v+\int_{S} \phi(\mathbf{x}, z)\left[\frac{\tilde{\psi}_{i}(\mathbf{x})-k(\mathbf{x}) \frac{\partial \tilde{\psi}_{i}(\mathbf{x})}{\partial \mathbf{n}}}{\alpha(\mathbf{x})+\beta(\mathbf{x})}\right] d s$,

transformed source term

For a general purpose and automatic implementation, it is quite desirable to employ a flexible computational approach to handle eigenvalue problems with arbitrarily variable coefficients, such as problem (3). Thus, the Generalized Integral Transform Technique (GITT) is here employed in the solution of the Sturm-Liouville problem (3) via the proposition of a simpler auxiliary eigenvalue problem, and expansion of the unknown eigenfunctions in terms of the chosen basis [19]. Also, the variable equation coefficients may themselves be expanded in terms of known eigenfunctions [13], so as to allow for a fully analytical implementation of the coefficients matrices in the transformed system. The equation coefficients of the auxiliary problem are simpler forms of the original coefficients, chosen so as to allow for an analytical solution of the auxiliary problem $[13,19]$. The boundary conditions types of the original and auxiliary problems are allowed to be different, in case further simplification of the auxiliary function is desired. Then, the resulting algebraic problem can be numerically solved to provide results for the eigenvalues and eigenvectors, which will be combined to provide the desired eigenfunctions of the original eigenvalue problem, as described in further detail in [13].

In order to illustrate both the direct and inverse problems solutions, we consider the two-dimensional situation of parallelplates micro-channels, with steady thermally developing laminar flow under the slip-flow regime. The fluid is assumed to enter the channel with a fully developed velocity profile and a uniform temperature, exchanging heat by convection with the surroundings with an external heat transfer coefficient that might not be known a priori in the inverse problem analysis. Thermophysical properties are assumed to be constant, while axial conduction and viscous dissipation are neglected.

Although more involved formulations could be handled by the proposed approach, the direct problem solution is here illustrated for the parallel-plates channel incompressible flow case, previously solved in [6,7] for the prescribed wall temperature boundary condition, and here written in a more general form including the external wall convection effect:

$$
\begin{aligned}
& W(Y) \frac{\partial \theta(Y, Z)}{\partial Z}=\frac{\partial^{2} \theta(Y, Z)}{\partial Y^{2}}, 0<Y<1, Z>0 \\
& \theta(Y, 0)=1, \quad 0 \leq Y \leq 1 \\
& \left.\frac{\partial \theta(Y, Z)}{\partial Y}\right|_{Y=0}=0,\left.\frac{\partial \theta(Y, Z)}{\partial Y}\right|_{Y=1}=-\frac{B i}{1+2 K n \beta_{t} B i} \theta(1, z), \quad Z>0
\end{aligned}
$$

where the corresponding dimensionless groups are given by

$Y=\frac{y}{y_{1}} ; Z=\frac{\alpha z}{u_{a v} y_{1}^{2}} ; \theta(Y, Z)=\frac{T(y, z)-T_{\infty}}{T_{0}-T_{\infty}} ;$

$W(Y)=\frac{u(y)}{u_{a v}} ; B i=\frac{h y_{1}}{k_{f}} ; K n=\frac{\lambda}{2 y_{1}}$

and,

$\beta_{t}=\frac{\left(2-\alpha_{t}\right)}{\alpha_{t}} \frac{2 \gamma}{(\gamma+1)} \frac{1}{\mathrm{Pr}}$

is the wall temperature jump coefficient and $\alpha_{t}$ is the thermal accommodation coefficient, $\lambda$ is the molecular mean free path, $\gamma=c_{p} / c_{v}$, while $c_{p}$ is specific heat at constant pressure, $c_{v}$ specific heat at constant volume and $\operatorname{Pr}$ is the Prandtl number. The dimensionless velocity profile is given as [6]:

$W(Y)=\frac{6 K n \beta_{v}+3\left(1-Y^{2}\right) / 2}{1+6 K n \beta_{v}}$

where,

$\beta_{v}=\frac{\left(2-\alpha_{m}\right)}{\alpha_{m}}$

is the wall velocity slip coefficient and $\alpha_{m}$ is the tangential momentum accommodation coefficient. The ratio of the boundary conditions coefficients is also of interest, and given as $\beta=\beta_{t} / \beta_{v}$.

The following correspondence of the forced convection problem (5) with the general formulation, eqs. (1), is then identified:

$Z \leftarrow Z, \quad \mathbf{x} \leftarrow Y, \quad w(\mathbf{x}) \leftarrow W(Y), \quad k(\mathbf{x}) \leftarrow 1, d(\mathbf{x}) \leftarrow 0$,

$P(\mathbf{x}, z) \leftarrow 0, f(\mathbf{x}) \leftarrow 1, \phi(\mathbf{x}, z) \leftarrow 0$,

$\alpha(\mathbf{x}) \leftarrow 0$ for $Y=0$ and $\frac{B i}{1+2 K n \beta_{t} B i}$ for $Y=1, \quad \beta(\mathbf{x}) \leftarrow 1$

The solution of the dimensionless problem (5) is then a special case from the general solution given in eq. (2), written as [6]:

$\theta(Y, Z)=\sum_{i=1}^{\infty} \bar{f}_{i} \tilde{\psi}_{i}(Y) e^{-\mu_{i}^{2} z}, \quad$ with $\bar{f}_{i}=-\frac{\tilde{\psi}_{i}^{\prime}(1)}{\mu_{i}^{2}}$

where $\psi_{i}(Y)$ are eigenfunctions of the following Sturm-Liouville problem, with the corresponding normalization integral and normalized form of the eigenfunction:

$\frac{\mathrm{d}^{2} \psi_{i}(Y)}{\mathrm{d} Y^{2}}+\mu_{i}^{2} W(Y) \psi_{i}(Y)=0, \quad 0<Y<1$

$\left.\frac{d \psi_{i}(Y)}{d Y}\right|_{Y=0}=0,\left.\quad \frac{d \psi_{i}(Y)}{d Y}\right|_{Y=1}=-\frac{B i}{1+2 K n \beta_{t} \beta i} \psi_{i}(1)$

$N_{i}=\int_{0}^{1} W(Y) \psi_{i}^{2}(Y) d Y ; \quad \tilde{\psi}_{i}(Y)=\frac{\psi_{i}(Y)}{N_{i}^{1 / 2}}$ 
The solution of problem (10) was obtained in [6,7] in terms of the confluent hypergeometric function, also known as Kummer function, readily available in the Mathematica system [32]. Here, a more convenient path for the corresponding inverse problem analysis was chosen, in light of the intensive computational task required by the parameters identification algorithm, so as to provide a solution in terms of simpler functions. Thus, following the ideas in the Generalized Integral Transform Technique, GITT [13,15,19], the solution of problem (10) is provided as an eigenfunction expansion from a simpler eigenvalue problem, that retains the same boundary conditions of the original problem, but avoids the variable coefficient corresponding to the dimensionless velocity field, in the form:

$\frac{d^{2} \Omega_{n}(Y)}{d Y^{2}}+\lambda_{n}^{2} \Omega_{n}(Y)=0, \quad 0<Y<1$

$\left.\frac{d \Omega_{n}(Y)}{d Y}\right|_{Y=0}=0,\left.\quad \frac{d \Omega_{n}(Y)}{d Y}\right|_{Y=1}=-\frac{B i}{1+2 K n \beta_{t} B i} \Omega_{n}(1)$

which is readily solved as

$\Omega_{n}(Y)=\cos \left(\lambda_{n} Y\right), \lambda_{n} \tan \left(\lambda_{n}\right)=\frac{B i}{1+2 K n \beta_{t} B i}, n=1,2, \ldots$

Once the auxiliary eigenfunctions and eigenvalues have been obtained, we may express the desired eigenfunction of the original problem as an expansion of these simpler functions:

$\psi_{i}(Y)=\sum_{n=1}^{\infty} \tilde{\Omega}_{n}(Y) \bar{\psi}_{i, n}, \quad$ inverse

$\bar{\psi}_{i, n}=\int_{0}^{1} \tilde{\Omega}_{n}(Y) \psi_{i}(Y) d Y, \quad$ transform

The integral transformation of the original eigenvalue problem is then performed by employing the operator $\int_{0}^{1} \tilde{\Omega}_{n}(Y)-d Y$, over eq. (10a), which results in the following algebraic eigenvalue problem for the original problem eigenvalues and corresponding eigenvectors:

$\left(\mathbf{A}-\mu^{2} \mathbf{B}\right) \bar{\psi}=0$

$\bar{\psi}=\left\{\bar{\psi}_{n, m}\right\} ; \mathbf{B}=\left\{B_{n, m}\right\}, B_{n, m}=\int_{0}^{1} W(Y) \tilde{\Omega}_{n}(Y) \tilde{\Omega}_{m}(Y) d Y$

$A_{n, m}=\lambda_{n}^{2} \delta_{n, m}, \quad$ where $\delta_{n, m}=1$, for $n=m$, or

$\delta_{n, m}=0$, for $n \neq m$

The algebraic problem (15) can be numerically solved to provide results for the eigenvalues $\mu^{2}$ and eigenvectors $\bar{\psi}$ from this matrix eigenvalue problem analysis [32] which will be combined within the inverse formula (14a) to provide the desired eigenfunctions of the original eigenvalue problem.

The fluid temperature along the wall is then directly computed from the inverse formula as:

$\theta(1, Z)=\sum_{i=1}^{\infty} \bar{f}_{i} \tilde{\psi}_{i}(1) e^{-\mu_{i}^{2} Z}$

and the corresponding temperature jump is accounted for so as to provide the temperature distribution at the wall itself:

$\theta_{w}(Z)=\frac{1}{1+2 \operatorname{KnBi} \beta_{t}} \theta(1, Z)$

The average temperature and the local Nusselt number based on the wall temperature at the interface, along the channel length, are then determined from: $\theta_{a v}(Z)=\sum_{i=1}^{\infty} \frac{\left(\tilde{\psi}_{i}^{\prime}(1)\right)^{2}}{\mu_{i}^{4}} e^{-\mu_{i}^{2} Z}$

$N u(Z)=\frac{\sum_{i=1}^{\infty} \frac{\left(\tilde{\psi}_{i}^{\prime}(1)\right)^{2}}{\mu_{i}^{2}} e^{-\mu_{i}^{2} Z}}{\theta_{a v}(Z)-\theta_{w}(Z)}$

\subsection{Inverse problem solution}

A variety of techniques is nowadays available for the solution of inverse problems [26,27]. However, one common approach relies on the minimization of an objective function that generally involves the squared difference between measured and estimated variables, like the least-squares norm, as well as some kind of regularization term. Despite the fact that the minimization of the least-squares norm is indiscriminately used, it only yields maximum likelihood estimates if the following statistical hypotheses are valid: the errors in the measured variables are additive, uncorrelated, normally distributed, with zero mean and known constant standard deviation; only the measured variables appearing in the objective function contain errors; and there is no prior information regarding the values and uncertainties of the unknown parameters.

Although very popular and useful in many situations, the minimization of the least-squares norm is a non-Bayesian estimator. A Bayesian estimator [28] is basically concerned with the analysis of the posterior probability density, which is the conditional probability of the parameters given the measurements, while the likelihood is the conditional probability of the measurements given the parameters. If we assume the parameters and the measurement errors to be independent Gaussian random variables, with known means and covariance matrices, and that the measurement errors are additive, a closed form expression can be derived for the posterior probability density. In this case, the estimator that maximizes the posterior probability density can be recast in the form of a minimization problem involving the maximum a posteriori objective function. On the other hand, if different prior probability densities are assumed for the parameters, the posterior probability distribution may not allow an analytical treatment. In this case, Markov Chain Monte Carlo (MCMC) methods are used to draw samples of all possible parameters, so that inference on the posterior probability becomes inference on the samples. In this work, we illustrate the use of Bayesian techniques for the estimation of parameters in micro-scale forced convection problems, via MCMC methods [28-31], as applied to the simultaneous identification of the momentum and thermal accommodation coefficients in slip-flow modeling. The Metropolis-Hastings algorithm is employed for the sampling procedure, implemented in the Mathematica platform [32].

Therefore, the unknown parameters in our formulation are given by:

$\mathbf{P}^{T}=\left[\beta_{v}, \beta_{t}, B i\right]$

Such unknown parameters were estimated in this work by using a Bayesian approach, as now described. Bayes' theorem can be stated as [28-30]:

$\pi_{\text {posterior }}(\mathbf{P})=\pi(\mathbf{P} \mid \mathbf{U})=\frac{\pi(\mathbf{P}) \pi(\mathbf{U} \mid \mathbf{P})}{\pi(\mathbf{U})}$

where $\pi_{\text {posterior }}(\mathbf{P})$ is the posterior probability density, that is, the conditional probability of the parameters $\mathbf{P}$ given the measurements $\mathbf{U} ; \pi(\mathbf{P})$ is the prior density, that is, a statistical model for the 
Table 1


$\mathrm{Bi}=\infty$, and reference results for $\mathrm{Bi}=1$.

\begin{tabular}{|c|c|c|c|c|c|c|c|}
\hline \multicolumn{4}{|c|}{$\mathrm{Bi}=\infty$} & \multicolumn{4}{|c|}{$\mathrm{Bi}=1.0$} \\
\hline Z & $\mathrm{Nu}(Z)$ & Z & $\mathrm{Nu}(Z)$ & $\mathrm{Z}$ & $\mathrm{Nu}(Z)$ & $\mathrm{Z}$ & $\mathrm{Nu}(Z)$ \\
\hline 0.01 & $2.702902 .70289^{*}$ & 0.06 & $1.836081 .83608^{*}$ & 0.01 & 2.81057 & 0.06 & 1.96128 \\
\hline 0.02 & $2.331122 .33111^{*}$ & 0.07 & $1.780711 .78071^{*}$ & 0.02 & 2.45481 & 0.07 & 1.90338 \\
\hline 0.03 & $2.130912 .13090^{*}$ & 0.08 & $1.736271 .73627^{*}$ & 0.03 & 2.25900 & 0.08 & 1.85615 \\
\hline 0.04 & $2.000272 .00027^{*}$ & 0.09 & $1.699961 .69996^{*}$ & 0.04 & 2.12882 & 0.09 & 1.81694 \\
\hline 0.05 & $1.906761 .90676^{*}$ & 0.1 & $1.669931 .66992^{*}$ & 0.05 & 2.03406 & 0.1 & 1.78394 \\
\hline
\end{tabular}

Corresponds to results of Ref. [6].

information about the unknown parameters prior to the measurements; $\pi(\mathbf{U} \mid \mathbf{P})$ is the likelihood function, which gives the relative probability density (loosely speaking, relative probability) of different measurement outcomes $\mathbf{U}$ with a fixed $\mathbf{P}$, and $\pi(\mathbf{U})$ is the marginal probability density of the measurements, which plays the role of a normalizing constant.

In this work we assume that the measurement errors are Gaussian random variables, with known (modeled) means and covariances, and that the measurement errors are additive and independent of the unknowns. With these hypotheses, the likelihood function can be expressed as [28-30]:

$\pi(\mathbf{U} \mid \mathbf{P})=(2 \pi)^{-M / 2}|\mathbf{W}|^{-1 / 2} \exp \left\{-\frac{1}{2}[\mathbf{U}-\Theta(\mathbf{P})]^{T} \mathbf{W}^{-1}[\mathbf{U}-\Theta(\mathbf{P})]\right\}$

where $M$ is the number of measurements, $\mathbf{W}$ is the covariance matrix of the measurement errors and $\boldsymbol{\Theta}(\mathbf{P})$ is the solution of the direct problem obtained with the vector of parameters $\mathbf{P}$. We note that parameters estimated through the maximization of eq. (19) are referred to as maximum likelihood estimates [26].

The unknown parameters in this study were estimated by using the Metropolis-Hastings algorithm for the Markov Chain Monte Carlo (MCMC) method [28-30], as described below. The implementation of the Metropolis-Hastings algorithm starts with the selection of a jumping distribution $p\left(\mathbf{P}^{*}, \mathbf{P}^{(t-1)}\right)$ which is used to draw a new candidate state $\mathbf{P}^{*}$, given the current state $\mathbf{P}^{(t-1)}$ of the Markov chain. Once the jumping distribution has been selected, the Metropolis-Hastings sampling algorithm can be implemented by repeating the following steps:

1. Sample a candidate point $\mathbf{P}^{*}$ from a jumping distribution $p\left(\mathbf{P}^{*}, \mathbf{P}^{(t-1)}\right)$.

2. Calculate the acceptance factor

$$
\alpha=\min \left[1-\frac{\pi\left(\mathbf{P}^{*} \mid \mathbf{U}\right) p\left(\mathbf{P}^{(t-1)}, \mathbf{P}^{*}\right)}{\pi\left(\mathbf{P}^{(t-1)} \mid \mathbf{U}\right) p\left(\mathbf{P}^{*}, \mathbf{P}^{(t-1)}\right)}\right]
$$

3. Generate a random value $u$ which is uniformly distributed on $[0,1]$.

4. If $u \leq \alpha$, set $\mathbf{P}^{t}=\mathbf{P}^{*}$; otherwise, set $\mathbf{P}^{t}=\mathbf{P}^{(t-1)}$.

5. Return to step 1 in order to generate the sequence $\left\{\mathbf{P}^{1}, \mathbf{P}^{2}, \ldots, \mathbf{P}^{n}\right\}$.

In this way, a sequence is generated to represent the posterior distribution and inference on this distribution is obtained from inference on the samples $\left\{\mathbf{P}^{1}, \mathbf{P}^{2}, \ldots, \mathbf{P}^{n}\right\}$. We note that values of $\mathbf{P}^{i}$ must be ignored until the chain has converged to equilibrium (the burn in period). For more details on theoretical aspects of the Metropolis-Hastings algorithm and MCMC methods, the reader should consult references [28-31].

\section{Results and discussion}

The direct problem solution is first validated by direct comparison with the benchmark results provided in [6], as illustrated in Table 1, for the case of parallel-plates under prescribed uniform wall temperature $(\mathrm{Bi}=\infty)$. The results in [6] were obtained from the classical integral transform method as well, but utilizing the exact solution of the related eigenvalue problem in terms of confluent hypergeometric functions, as obtained from a symbolic computation platform [32]. The numerical results for the local Nusselt number along the channel length obtained from the two approaches are practically coincident to the six significant digits presented. The Nusselt number results for the case of $\mathrm{Bi}=1$ here computed are also presented for reference purposes.

Next, the inverse problem solution is illustrated, by adopting the following typical values of the governing parameters as indicated in



Fig. 1. (a) Influence of Biot number on the sensivity matrix determinant in terms of the number of measurements along the channel for the simultaneous estimation of the three parameters $(\mathrm{Bi}=0.1$, solid line; $\mathrm{Bi}=1$, short dashes; $\mathrm{Bi}=10$, long dashes). (b) Comparison of reduced sensitivity coefficients in terms of the number of measurements along the channel for the three parameters with $\mathrm{Bi}=1\left(\beta_{v}\right.$ in short dashes, $\beta_{t}$ in solid line, $\mathrm{Bi}$ in long dashes). 
Table 2

Estimated parameters values with 50,000 states in Markov chains (neglecting first 10,000 states for the chains burn in) and the corresponding $95 \%$ confidence intervals for the base case.

\begin{tabular}{lllllll}
\hline Parameter & Exact & Initial & Interval & Estimated & Min. with 95\% & Max. with 95\% \\
\hline$\beta_{v}$ & 1.5 & 3 & {$[1,5]$} & 1.518 & 1.308 & 1.789 \\
$\beta_{t}$ & 2.0 & 3 & {$[1,5]$} & 2.010 & 1.876 & 2.144 \\
$\mathrm{Bi}$ & 1.0 & 5.05 & {$[0.1,10]$} & 1.000 & 0.996 & 1.003 \\
\hline
\end{tabular}

ref. [24], $\beta_{v}=1.5, \beta_{t}=2.0, \mathrm{Kn}=0.025$, and $\mathrm{Bi}=1.0$. Only wall temperature measurements, obtained from simulated data as computed from eq. (16b), are employed in this work. A total of 1000 uniformly distributed points along the dimensionless channel length, $Z_{f}=5$, was initially adopted. The simulated measurements were considered normally distributed with averages at the simulated values and $1 \%$ standard deviation. They were obtained with 50 terms in the eigenfunction expansions, while the direct problem solution within the inverse problem procedure was handled with 20 terms only, in order to avoid the so called inverse crime [28].

Before addressing the estimation of the unknown parameters, the behavior of the determinant of the information matrix $\mathbf{J}^{T} \mathbf{J}$ $[26,27]$ was analyzed in order to inspect the influence of the parameters to be estimated in the solution of the inverse problem. The sensitivity matrix $\mathbf{J}$ is defined as:



The sensitivity coefficients $J_{i j}=\partial T_{i} / \partial P_{j}$ give the sensitivity of $T_{i}$ (solution of the direct problem) with respect to changes in the parameter $P_{j}$. A small value of the magnitude of $j_{i j}$ indicates that large changes in $P_{j}$ yield small changes in $T_{j}$. It can be said that the estimation of the parameter $P_{j}$ is extremely difficult in such cases, because basically the same value for $T_{i}$ would be obtained for a wide range of values of $P_{j}$. In fact, when the sensitivity coefficients are small, $\left|\mathbf{J}^{T} \mathbf{J}\right| \approx 0$ the inverse problem is ill-conditioned. It can also be shown that $\left|\mathbf{J}^{T} \mathbf{J}\right|$ is null if any column of $\mathbf{J}$ can be expressed as a linear combination of other columns [26]. Therefore, it is desirable to have linearly-independent sensitivity coefficients $J_{\mathrm{ij}}$ with large magnitudes, so that the parameter estimation problem is not very sensitive to measurement errors and accurate estimates of the parameters can be obtained. The comparison of the magnitude of the sensitivity coefficients, as well as the analysis of possible linear dependence, is more easily performed by using the reduced sensitivity coefficients instead of the original ones. The reduced sensitivity coefficients are obtained by multiplying the original sensitivity coefficients, $J_{\mathrm{ij}}$, by the parameters that they refer to. Therefore, they have units of the measured variables, which are used as a basis of comparison.

Based on possible experimental setups, we will consider three different orders of magnitude of the Biot number $(B i=0.1,1$ and 10$)$ for the analysis of the determinant of the information matrix (Fig. 1a). Clearly, by increasing the Biot number to $\mathrm{Bi}=10$, markedly decreases the value of the determinant. Also, for the two lower values of $\mathrm{Bi}$, it has been observed that the determinants are indeed larger, with a still increasing value for the lower value $\mathrm{Bi}=0.1$, and an almost stabilized value for the intermediate Biot number, $\mathrm{Bi}=1.0$, at the end of the channel at $Z_{f}=5$. Fig. 1b illustrates the reduced sensitivity coefficients for each of the three parameters obtained with $\mathrm{Bi}=1.0$. This figure indicates that the estimation of the Biot number should not pose difficulties, because its sensitivity coefficient is large and linearly independent with respect to the others. On the other hand, the sensitivity coefficients with respect to the two accommodation coefficients are small and linearly dependent, so that the simultaneous estimation of these two parameters may not be possible, unless an informative prior is provided for at least one of them. In fact, an initial attempt of estimating the three parameters was performed by providing a priori information in the form of uniform probability distribution functions for all three parameters, within the admissible minimum and maximum values intervals for each parameter. It was then observed that, especially for the parameter $\beta_{v}$, more informative prior would be required for achieving convergence in the estimation procedure. Fortunately, a priori information for both $\beta_{v}$ and Bi can in principle be obtained for

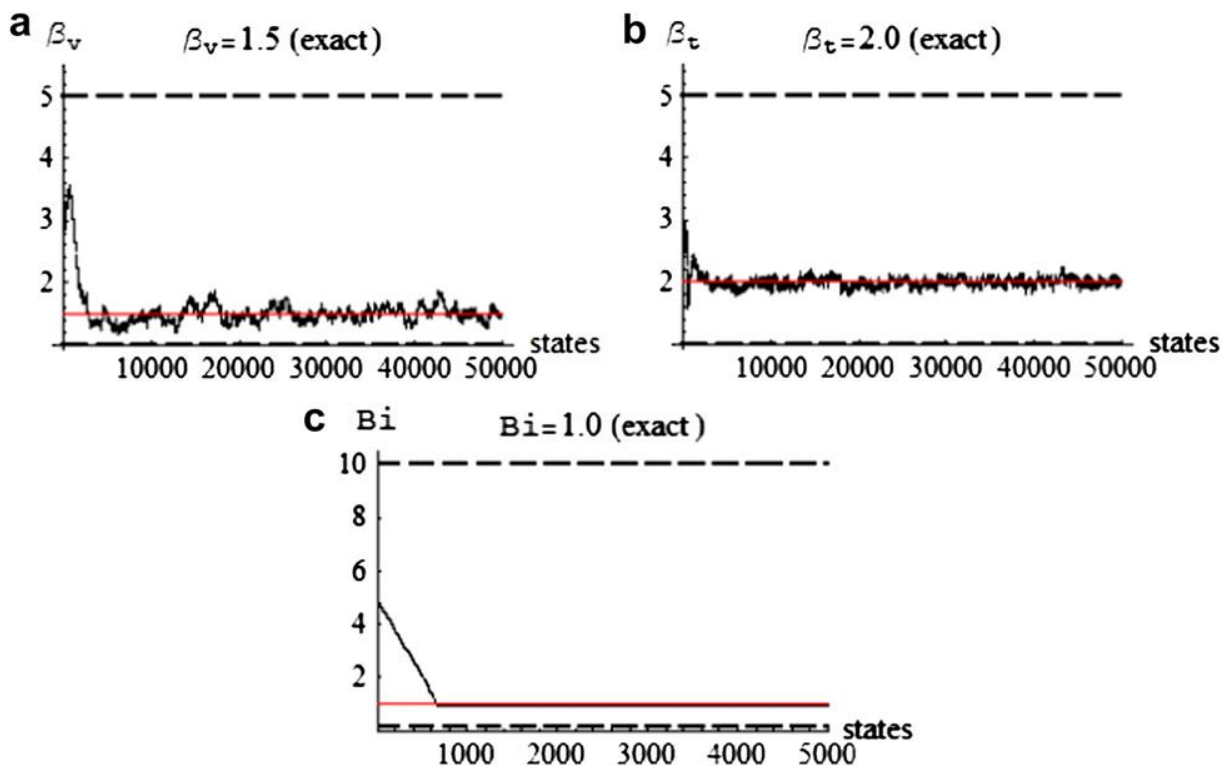

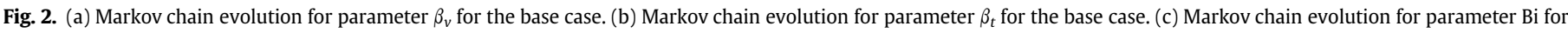
the base case. 
a

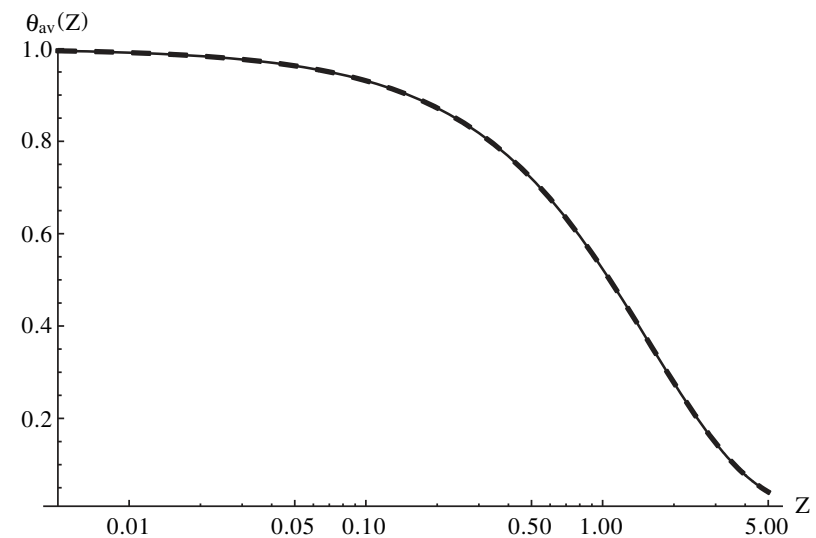

b $\mathrm{Nu}(\mathrm{Z})$

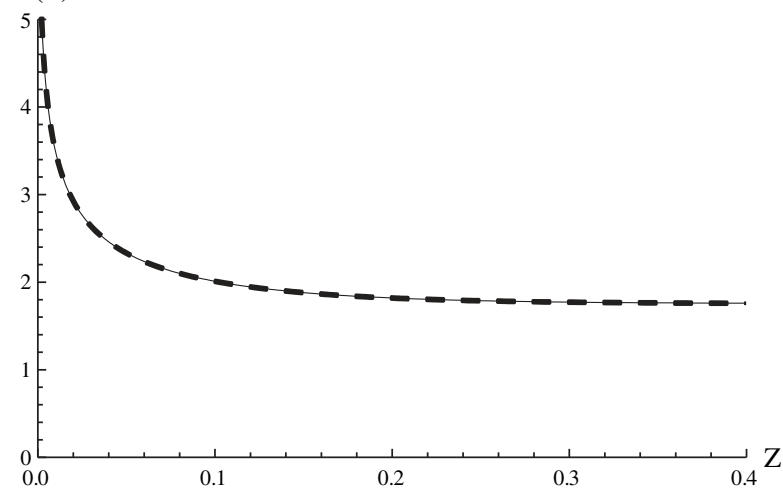

Fig. 3. (a) Bulk temperature distribution as computed with exact (solid line) and estimated (dashed thick line) parameters for the base case. (b) Local Nusselt number distribution as computed with exact (solid line) and estimated (dashed thick line) parameters for the base case.
Table 3

Estimated parameters values with 50,000 states in Markov chains (neglecting first 10,000 states for the chains burn in) and the corresponding $95 \%$ confidence intervals for different initial guesses (equal to minimum limits).

\begin{tabular}{lllllll}
\hline Parameter & Exact & Initial & Interval & Estimated & $\begin{array}{l}\text { Min. } \\
\text { with 95\% }\end{array}$ & $\begin{array}{l}\text { Max. } \\
\text { with 95\% }\end{array}$ \\
\hline$\beta_{v}$ & 1.5 & 1 & {$[1,5]$} & 1.539 & 1.305 & 1.803 \\
$\beta_{t}$ & 2.0 & 1 & {$[1,5]$} & 2.011 & 1.870 & 2.143 \\
$\mathrm{Bi}$ & 1.0 & 0.1 & {$[0.1,10]$} & 1.000 & 0.997 & 1.003 \\
\hline
\end{tabular}

most experimental conditions, by utilizing pressure and mass flow rate measurements to approximate the slip coefficient and by employing classical correlations for estimating the external heat transfer coefficient, respectively. We have then proceeded to the analysis of the inverse problem by providing normal probability distributions as priors for these two parameters $\left(\beta_{v}\right.$ and $\left.\mathrm{Bi}\right)$, while maintaining the uniform probability distribution as prior for $\beta_{t}$ in the interval $[1,5]$. The Gaussian priors for $\beta_{v}$ and Bi were initially assumed with means at the exact values and $10 \%$ standard deviations. However, larger standard deviations were also examined in order to further challenge the convergence behavior of the Markov chains, as described below.

We first illustrate the Markov chains for each of the three parameters for the base case, involving Gaussian priors for $\beta_{v}$ and Bi with $10 \%$ standard deviations. In this case, the chains were started from the average values between the admissible minimum and maximum limits (Table 2). Fig. 2a-c illustrate the evolution of the Markov chains (up to 50,000 states) for the estimation of the three parameters, $\beta_{v}, \beta_{t}$, and $\mathrm{Bi}$, respectively. Also shown in these figures are the straight lines that correspond to the admissible minimum and maximum limits for each specific parameter, while the initial values were taken as the averages of these two values. One may clearly observe that the Markov chain for the parameter Bi has a distinguished behavior of a very fast convergence, in comparison to the other two parameters, requiring less than 1000 states and for this reason this particular plot is reduced to only 5000 states to allow for this verification. The slip boundary condition coefficient, $\beta_{v}$, appears
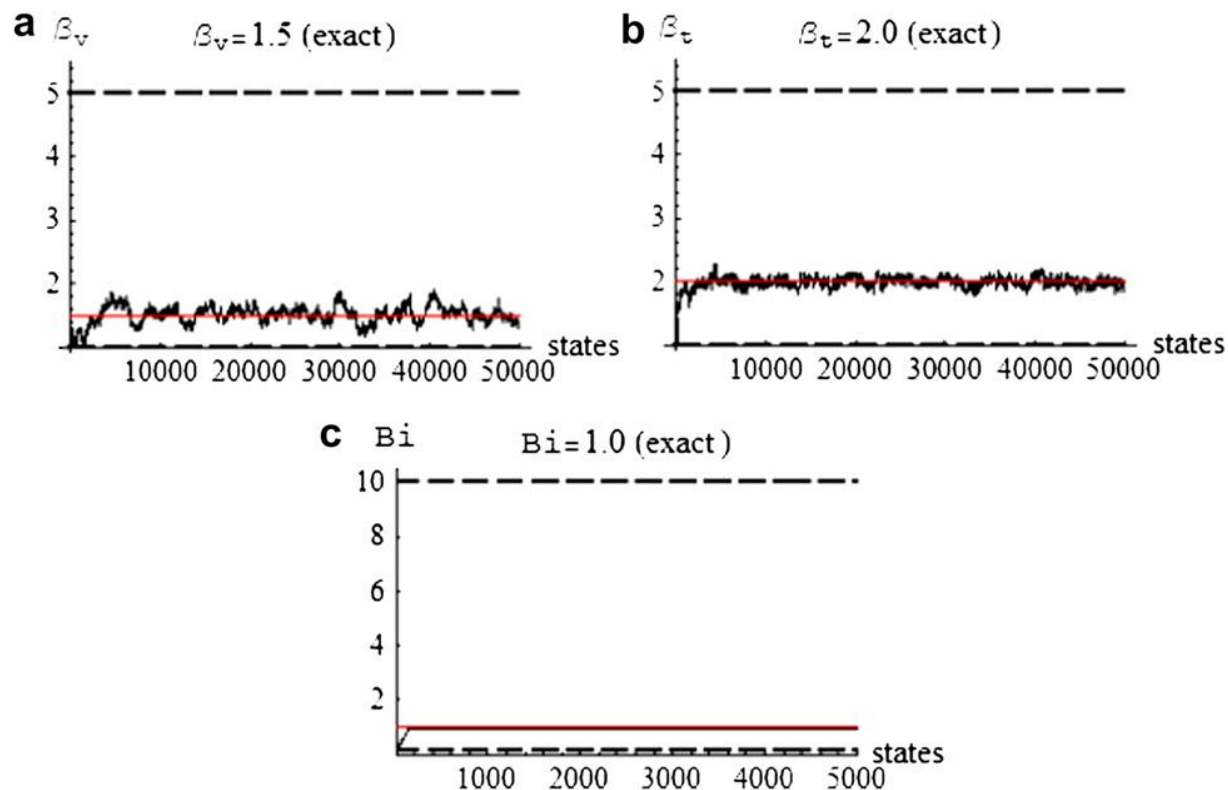

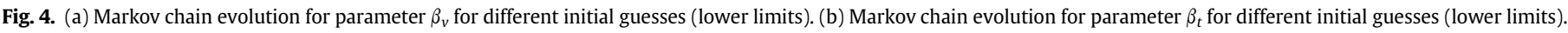
(c) Markov chain evolution for parameter Bi for different initial guesses (lower limits). 




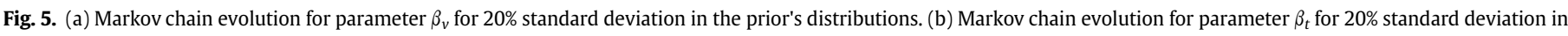
the prior's distributions. (c) Markov chain evolution for parameter Bi for 20\% standard deviation in the prior's distributions.

Table 4

Estimated parameters values with 50,000 states in Markov chains (neglecting first 10,000 states for the chains burn in) and the corresponding $95 \%$ confidence intervals for $20 \%$ standard deviation in the priors distributions.

\begin{tabular}{lllllll}
\hline Parameter & Exact & Initial & Interval & Estimated & $\begin{array}{l}\text { Min. } \\
\text { with 95\% }\end{array}$ & $\begin{array}{l}\text { Max. } \\
\text { with 95\% }\end{array}$ \\
\hline$\beta_{v}$ & 1.5 & 3 & {$[1,5]$} & 1.629 & 1.187 & 2.110 \\
$\beta_{t}$ & 2.0 & 3 & {$[1,5]$} & 2.046 & 1.850 & 2.234 \\
$\mathrm{Bi}$ & 1.0 & 5.05 & {$[0.1,10]$} & 1.000 & 0.997 & 1.003 \\
\hline
\end{tabular}

to be the most difficult one to estimate in the present situation, as also indicated by the respective sensitivity analysis (Fig. 1b). Table 2 summarizes the input data and illustrates not only the estimated values, after neglecting the first 10,000 states in each chain, but also the minimum and maximum values of the $95 \%$ confidence intervals for such estimated parameters. Even for the least sensitive parameter, $\beta_{v}$, the exact value lies within the confidence intervals, though it presents the widest interval among the three estimated parameters. Fig. 3a,b illustrates the excellent agreement of the bulk temperature and local Nusselt number distributions, respectively, as obtained with the exact and estimated values for the parameters, with the exact curves in solid thin lines and the curves obtained with the estimated parameters drawn with dashed thicker lines.
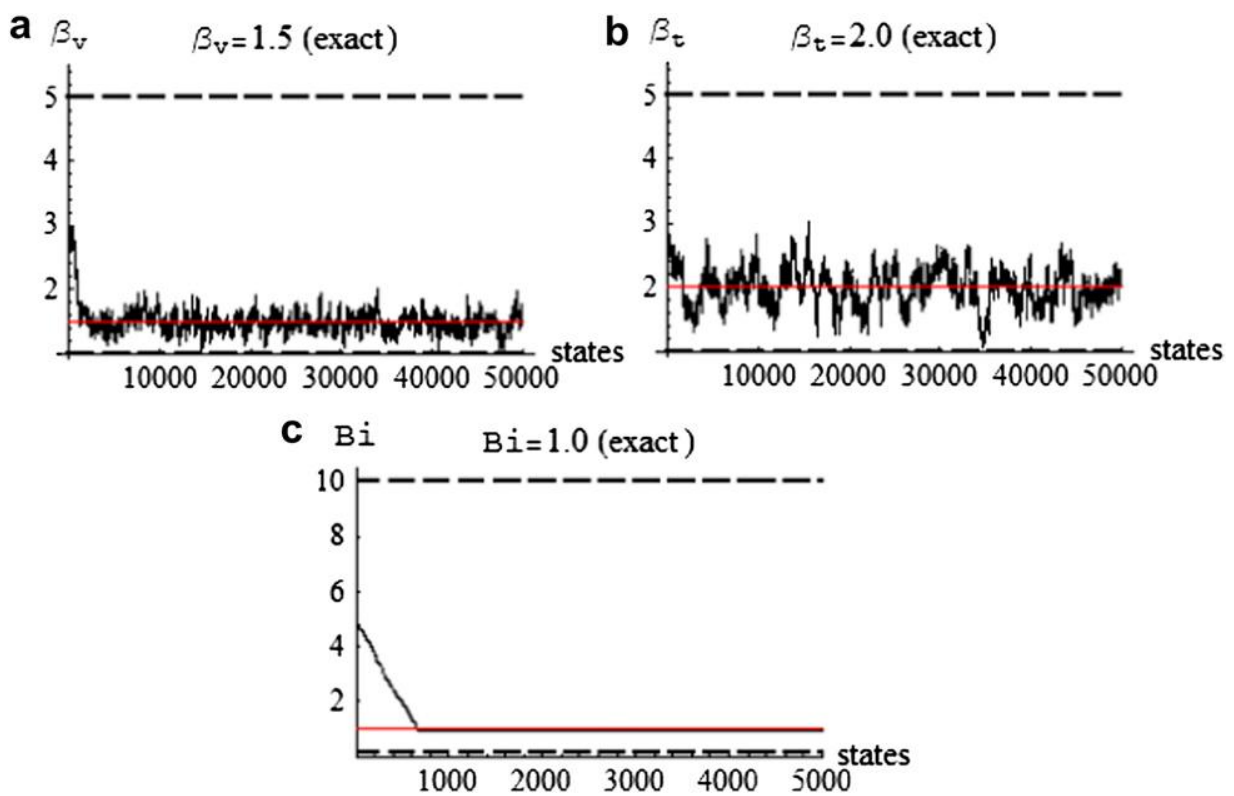

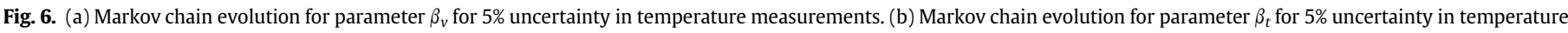
measurements. (c) Markov chain evolution for parameter Bi for $5 \%$ uncertainty in temperature measurements. 
Table 5

Estimated parameters values with 50,000 states in Markov chains (neglecting first 10,000 states for the chains burn in) and the corresponding $95 \%$ confidence intervals for $5 \%$ uncertainty in temperature measurements.

\begin{tabular}{lllllll}
\hline Parameter & Exact & Initial & Interval & Estimated & $\begin{array}{l}\text { Min. } \\
\text { with 95\% }\end{array}$ & $\begin{array}{l}\text { Max. } \\
\text { with 95\% }\end{array}$ \\
\hline$\beta_{v}$ & 1.5 & 3 & {$[1,5]$} & 1.483 & 1.223 & 1.779 \\
$\beta_{t}$ & 2.0 & 3 & {$[1,5]$} & 2.001 & 1.430 & 2.557 \\
$\mathrm{Bi}$ & 1.0 & 5.05 & {$[0.1,10]$} & 1.001 & 0.983 & 1.019 \\
\hline
\end{tabular}

The next case considered is aimed at analyzing the effect of quite different initial guesses for the three parameters, and as an extreme choice, the minimum admissible values were employed for all of them. Fig. $4 a-c$ illustrate the evolution of the Markov chains (up to 50,000 states) for the estimation of the three parameters, with the same input data as the base case, but with such different starting points. The chains again show an excellent convergence behavior with the estimated values and corresponding confidence intervals shown in Table 3, which are very close to those achieved in the base case previously shown.

Then, we attempt to illustrate the effect of increasing the standard deviation of the average values informed as priors for the two coefficients $\beta_{v}$ and $\mathrm{Bi}$, now markedly increased to $20 \%$, returning to the initial guesses provided by the average values of the admissible limits as for the base case. From Fig. $5 a-c$, we can notice that the convergence of the Markov chains has now been noticeably affected, especially for the slip coefficients $\beta_{v}$ and $\beta_{t}$. For $\beta_{v}$ it is apparent that the burn in period seems to require a larger number of states (around 25,000 in this example). This behavior is also evident from the worst estimated values in Table 4, together with the wider confidence intervals, especially for the slip boundary condition coefficients.

Another variation of the base case is considered now by increasing the uncertainty of the simulated temperature measurements to $5 \%$, while returning to the Gaussian priors for $\beta_{v}$ and Bi with means at the exact values and $10 \%$ standard deviations. It is interesting to observe from Fig. $6 \mathrm{a}-\mathrm{c}$ that the estimation of $\beta_{v}$ is not markedly affected by
Table 6

Estimated parameters values with 50,000 states in Markov chains (neglecting first 10,000 states for the chains burn in) and the corresponding $95 \%$ confidence intervals for a total of 100 temperature measurements.

\begin{tabular}{lllllll}
\hline Parameter & Exact & Initial & Interval & Estimated & $\begin{array}{l}\text { Min. } \\
\text { with 95\% }\end{array}$ & $\begin{array}{l}\text { Max. } \\
\text { with 95\% }\end{array}$ \\
\hline$\beta_{v}$ & 1.5 & 3 & {$[1,5]$} & 1.474 & 1.211 & 1.757 \\
$\beta_{t}$ & 2.0 & 3 & {$[1,5]$} & 1.972 & 1.529 & 2.400 \\
$\mathrm{Bi}$ & 1.0 & 5.05 & {$[0.1,10]$} & 1.000 & 0.989 & 1.011 \\
\hline
\end{tabular}

increasing the measurement errors. On the other hand, the convergence behavior of the chain for $\beta_{t}$ is clearly altered, with larger amplitudes of oscillation, though still showing a convergence pattern. The Biot number is again the least sensible parameter to the uncertainty of the measurements, with an excellent convergence behavior in its Markov chain. Table 5 also reflects such facts, with a larger confidence interval for the temperature jump coefficient, $\beta_{t}$.

Finally, we examine the influence of reducing the number of experimental measurements by one order of magnitude, bringing the total number down to 100 points along the channel wall, as might be eventually required by resolution limitations of the specific thermographic equipment with respect to the microchannel length (see Fig. 7a-c). In this case, it has also been assumed to have Gaussian priors for $\beta_{v}$ and Bi with $10 \%$ standard deviations. After inspecting Fig. $7 \mathrm{a}-\mathrm{c}$, one may conclude that the $\beta_{t}$ Markov chain convergence is the one most noticeably affected, in comparison to the base case where a total of 1000 measurement points have been made available. Clearly, larger amplitudes on the $\beta_{t}$ Markov chain oscillations can be observed, while the Markov chains for the other two parameters do not present an evident variation with respect to those presented in Fig. 2a-c. The reduced number of measurement points similarly affects the width of the confidence interval for the parameter $\beta_{t}$, but again the estimated value remains quite reasonable, as observed from Table 6 . The estimation of the other two parameters is less affected by the reduction of experimental observations.


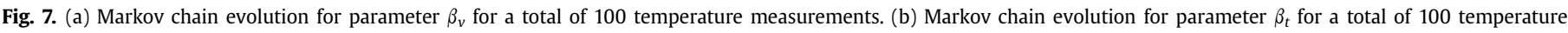
measurements. (c) Markov chain evolution for parameter Bi for a total of 100 temperature measurements. 


\section{Conclusions}

Inverse problem analysis of laminar forced convection within micro-channels has been undertaken, focusing on the identification of the momentum and thermal accommodation coefficients for gas flow within the slip-flow regime, together with the usually unknown Biot number for the walls heat exchange with the external environment. The aim is to demonstrate computational tools for the simultaneous estimation of these critical parameters in modeling heat and fluid flow in micro-channels, under actual operating conditions of the associated micro-systems. Also, the idealized experimental setup is based solely on temperature measurements of the external wall surface, as obtainable by infrared thermographic measurements. The Integral Transform method was employed in the direct problem solution, avoiding cumbersome functional representations in the original eigenfunction expansions by adopting a hybrid numerical-analytical solution for the related eigenvalue problem, implemented via the Generalized Integral Transform Technique (GITT). This combination of approaches provides a robust, precise and computationally fast solution of the direct problem, as required for the intensive computational inverse analysis.

A Bayesian approach of parameter estimation is applied and the unknown parameters are estimated with a Markov Chain Monte Carlo (MCMC) method, through the implementation of the MetropolisHastings algorithm. The Bayesian approach permits to rigorously take into account $a$ priori information available for the parameters, such as from previous experimental runs, or even from other experimental setups or theoretical predictions. Thus, a priori information usually available for the slip-flow boundary condition coefficient and for the Biot number are employed in the proposed estimation procedure, here considered in the form of Gaussian distributions. For the other parameter, the temperature jump boundary condition coefficient, a non-informative uniform distribution was used as prior. The results obtained with simulated measurements containing Gaussian random errors revealed the accuracy and robustness of the present estimation approach.

\section{Acknowledgements}

The authors would like to acknowledge the partial financial support provided by CNPq, CAPES and FAPERJ, Brazilian agencies for the fostering of sciences.

\section{References}

[1] P. Tabeling, Introduction a la Microfluidique, Belin, Collection Échelles, Paris, 2003.

[2] G. Karniadakis, A. Beskok, N. Aluru, Microflows and Nanoflows: Fundamentals and Simulation. Springer, NY, 2005.

[3] Y. Yener, S. Kakaç, M.R. Avelino, T. Okutucu, Single-phase forced convection in micro-channels - a state-of-the-art review. in: S. Kakaç, L.L. Vasiliev, Y. Bayazitoglu, Y. Yener (Eds.), Microscale Heat Transfer - Fundamentals and Applications, NATO ASI Series. Kluwer Academic Publishers, Netherlands, 2005, pp. 1-24.
[4] R.W. Barber, D.R. Emerson, Challenges in modeling gas-phase flow in microchannels: from slip to transition. Heat Transfer Eng. 27 (4) (2006) 3-12.

[5] C.B. Sobhan, G.P. Peterson, Microscale and Nanoscale Heat Transfer: Fundamentals and Engineering Applications. CRC Press, Boca Raton, FL, 2008.

[6] M.D. Mikhailov, R.M. Cotta, Mixed symbolic-numerical computation of convective heat transfer with slip flow in microchannels. Int. Commun. Heat Mass Transfer 32 (3-4) (2005) 341-348.

[7] R.M. Cotta, M.D. Mikhailov, S. Kakaç, Steady and periodic forced convection in microchannels. in: S. Kakaç, et al. (Eds.), NATO Science Series II: Microscale Heat Transfer: Fundamentals and Applications, vol. 193, 2005, pp. 49-74.

[8] S. Yu, T.A. Ameel, Slip flow heat transfer in rectangular microchannels. Int. J. Heat Mass Transfer 44 (2001) 4225-4234.

[9] G. Tunc, Y. Bayazitoglu, Heat transfer in microtubes with viscous dissipation. Int. J. Heat Mass Transfer 44 (2001) 2395-2403.

[10] G. Tunc, Y. Bayazitoglu, Heat transfer in rectangular microchannels. Int. J. Heat Mass Transfer 45 (2002) 765-773.

[11] F.V. Castellões, R.M. Cotta, Analysis of transient and periodic convection in micro-channels via integral transforms. Prog. Computat. Fluid Dynamics 6 (2006) 321-326.

[12] F.V. Castellões, C.R. Cardoso, P. Couto, R.M. Cotta, Transient analysis of slip flow and heat transfer in microchannels. Heat Transfer Eng. 28 (2007) 549-558.

[13] C.P. Naveira-Cotta, R.M. Cotta, H.R.B. Orlande, O. Fudym, Eigenfunction expansions for transient diffusion in heterogeneous media. Int. J. Heat Mass Transfer 52 (2009) 5029-5039.

[14] M.D. Mikhailov, M.N. Ozisik, Unified Analysis and Solution of Heat and Mass Diffusion. John Wiley, NY, 1984, also, Dover Publications, 1994.

[15] R.M. Cotta, Integral Transforms in Computational Heat and Fluid Flow. CRC Press, USA, 1993.

[16] R.M. Cotta, M.D. Mikhailov, Heat Conduction: Lumped Analysis, Integra Transforms, Symbolic Computation. Wiley-Interscience, NY, 1997.

[17] R.M. Cotta, The Integral Transform Method in Thermal and Fluids Sciences and Engineering. Begell House, New York, 1998.

[18] R.M. Cotta, M.D. Mikhailov, Hybrid methods and symbolic computations. in: W.J. Minkowycz, E.M. Sparrow, J.Y. Murthy (Eds.), Handbook of Numerical Heat Transfer, second ed. Wiley, New York, 2006, pp. 493-522.

[19] M.D. Mikhailov, R.M. Cotta, Integral transform method for eigenvalue problems. Commun. Numer. Methods. Eng. 10 (1994) 827-835.

[20] M.C. Oliveira, R. Ramos, R.M. Cotta, On the eigenvalues basic to the analytical solution of convective heat transfer with axial diffusion effects. Commun. Numer. Methods. Eng. 11 (1995) 287-296.

[21] L.A. Sphaier, R.M. Cotta, Integral transform analysis of multidimensional eigenvalue problems within irregular domains. Numer. Heat Transfer, Part B-Fundamentals 38 (2000) 157-175.

[22] A. Agrawal, S.V. Prabhu, Survey on measurement of tangential momentum accommodation coefficient. J. Vac. Sci. Technol. A 26 (4) (2008) 634-645.

[23] E.B. Arkilic, K.S. Breuer, M.A. Schmidt, Mass flow and tangential momentum accommodation in silicon micromachined channels. J. Fluid Mech. 437 (2001) 29-43.

[24] S.S. Hsieh, H.H. Tsai, C.Y. Lin, C.F. Huang, C.M. Chien, Gas flow in a long microchannel. Int. J. Heat Mass Transfer 47 (2004) 3877-3887.

[25] D.J. Rader, W.M. Trott, J.R. Torczynski, J.N. Castañeda, T.W. Grasser, Measurements of Thermal Accommodation Coefficients Report SAND2005-6084. Sandia National Laboratories, Albuquerque, 2005.

[26] J. Beck, K. Arnold, Parameter Estimation in Engineering and Science. Wiley Interscience, New York, 1977.

[27] M.N. Ozisik, H.R.B. Orlande, Inverse Heat Transfer: Fundamentals and Applications. Taylor and Francis, New York, 2000.

[28] J. Kaipio, E. Somersalo, Statistical and Computational Inverse Problems. Springer-Verlag, 2004

[29] P. Lee, Bayesian Statistics. Oxford University Press, London, 2004.

[30] D. Gamerman, H.F. Lopes, Markov Chain Monte Carlo: Stochastic Simulation for Bayesian Inference, second ed. Chapman \& Hall/CRC, Boca Raton, FL, 2006.

[31] O. Fudym, H.R.B. Orlande, M. Bamford, J.C. Batsale, Bayesian approach for thermal diffusivity mapping from infrared images processing with spatially random heat pulse heating. J. Phys. 135 (2008) 012-042 Conference Series (Online).

[32] S. Wolfram, The Mathematica Book version 7.0. Cambridge-Wolfram Media, 2008. 